\title{
Towards a VO compliant ESO science archive
}

\author{
Paolo Padovani, on behalf of the Virtual Observatory Systems \\ department at ESO
}

\author{
European Southern Observatory, Karl-Schwarzschild-Straße 2, \\ D-85748 Garching bei München, Germany \\ email: ppadovan@eso.org
}

Data centres have a major role in the Virtual Observatory (VO), as they are the primary source of astronomical data. The VO cannot (and does not) dictate how a data centre handles its own archive. However, 'VO-layer' is needed to 'translate' any locally defined parameter to the standard (i.e., International Virtual Observatory Alliance compliant) ones. The longer term vision of the VO is also to hide away any observatory/telescope/instrument specific detail and work in astronomical units, for example, 'wavelength range' and not grism or filter name. Data providers are then advised to systematically collect metadata ('data about data') about the curation process, assign unique identifiers, describe the general content (e.g., physical coverage) of a collection, and provide interface and capability parameters of public services. Finally, the VO will work at its best with high-level ('science-ready') data, so that the VO user is spared as much as possible any complex and time consuming data reduction. Data centres should then make an effort to provide such data.

All these issues affect also the European Southern Observatory, which operates one of the largest largest astronomical archives in the world. This currently holds more than 100 Terabytes of data but is predicted to increase its size $\sim 16$ times in the next 6 years. The Data Management and Operations Division has then created the Virtual Observatory Systems (VOS) Department on November 1, 2004. The role of VOS is to manage ESO's involvement in VO activities, but above all to make the Science Archive Facility (SAF) into a powerful scientific resource for the ESO community. The new department includes, at present, the Virtual Observatory Technology (VOT) and the Advanced Data Products (ADP) groups and is made up of fifteen people.

Current/recent SAF/VO activities include: an improved archive interface, which represents a first step towards a VO-compliant interface(<http://archive.eso.org >); sciencedriven requirements already implemented into the ESO metadata repository and data models to serve both the real and virtual observatory; the creation of ADPs, that is 'science-ready' data; and preparation for ADP ingestion and publication. ADPs currently scattered around the community are also being collected, validated and published into the SAF (according to detailed guidelines). ESO, in fact, now requests Large Programs and the upcoming VST and Vista surveys to provide their reduced data at the time of publication. This represents a paradigm change and underscores the fact that the investigators of an observing program are in the best position to analyze and provide properly reduced data. Science-ready imaging ADPs are also being produced in VOS by the ADP/MVM data reduction system. Based on the EIS/MVM software, this is aimed at ISAAC, WFI, SOFI and other VLT imaging instruments ADP production and was used for the reduction of the ISAAC/GOODS ADPs, released on September 30, 2005, which cover the deepest large near-IR field carried out to date.

Our VO plans for the next two years include: more ADPs (internal and above all from ESO PIs), a brand new VO-compliant archive interface (based on metadata registry), VO tools to enhance the archive interface, and the ingestion of Quality Control products. 